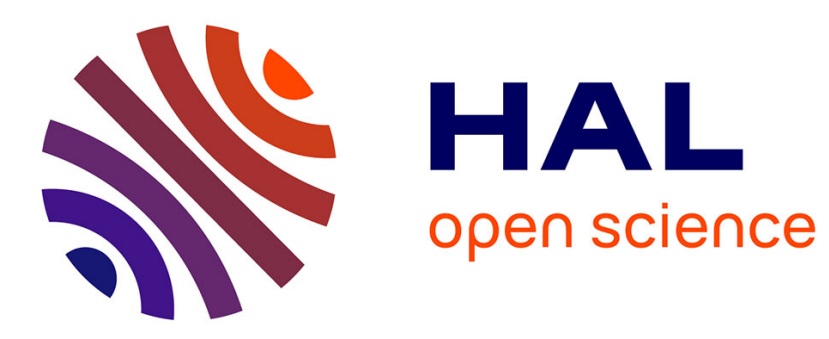

\title{
Understanding the omnidirectional capability of a generic multi-rotor aerial vehicle
}

\author{
Mahmoud Hamandi, Quentin Sable, Marco Tognon, Antonio Franchi
}

\section{To cite this version:}

Mahmoud Hamandi, Quentin Sable, Marco Tognon, Antonio Franchi. Understanding the omnidirectional capability of a generic multi-rotor aerial vehicle. IEEE Workshop on Aerial Robotic Systems Physically Interacting with the Environment (AIRPHARO), Oct 2021, Biograd na Moru, Croatia. 10.1109/AIRPHARO52252.2021.9571051 . hal-03402718

\section{HAL Id: hal-03402718 \\ https://hal.science/hal-03402718}

Submitted on 26 Oct 2021

HAL is a multi-disciplinary open access archive for the deposit and dissemination of scientific research documents, whether they are published or not. The documents may come from teaching and research institutions in France or abroad, or from public or private research centers.
L'archive ouverte pluridisciplinaire HAL, est destinée au dépôt et à la diffusion de documents scientifiques de niveau recherche, publiés ou non, émanant des établissements d'enseignement et de recherche français ou étrangers, des laboratoires publics ou privés. 


\title{
Understanding the omnidirectional capability of a generic multi-rotor aerial vehicle
}

\author{
Mahmoud Hamandi ${ }^{1}$, Quentin Sable ${ }^{2}$, Marco Tognon ${ }^{3}$ and Antonio Franchi ${ }^{2,1}$
}

\begin{abstract}
The aim of this work is to present the necessary conditions for the design of an omnidirectional Multi-Rotor Aerial Vehicle (MRAV), while taking into consideration its geometry, weight, and actuation limits. The work formally defines these conditions and presents numerical metrics that reflect the satisfaction of the omnidirectional property. These metrics are then applied to assess the omnidirectional property of "Omni-plus-seven", i.e., an omnidirectional MRAV consisting of a hepta-rotor with uni-directional thrusters [1]. Finally the work shows the use of such metrics in the design of a new platform with similar geometry and modified weight and actuators.
\end{abstract}

\section{INTRODUCTION}

Multi-Rotor Aerial Vehicles (MRAVs) have gained an increased popularity in the literature in the last few years [2]. This popularity has been coupled with applications in the fields of search and rescue, firefighting, human-robot interaction and aerial physical interaction. With the introduction of these applications, MRAVs were required to apply forces and moments in directions that are not possible with classical underactuated platforms. Thus, omnidirectional platforms resulted to be more suited for applications involving aerial physical interaction [3].

While the omnidirectional capability of a platform can be understood intuitively, we define it in the scope of this paper as the platform's ability to hover in any direction assuming it is lightweight enough. From an actuation point of view, it is the platform's ability to apply any force, in a sphere centered at the platform's Center of Mass (CoM), while applying any moment in the neighborhood of the zero-moment, decoupled from the applied force.

Multiple works in the literature demonstrated working omnidirectional prototypes, with each one studying a different design aspect of these platforms. Each of these prototypes relied on a different actuation strategy to achieve omnidirectional flight. The authors in [4] presented one of the first omnidirectional prototypes, where they placed eight bidirectional propellers on the edges of an octahedron. One of the novelties of their design is the optimization of the placement of the propellers, where the octahedron shape was chosen to ensure a rotation-invariant inertia tensor. Another

${ }^{1}$ LAAS-CNRS, Université de Toulouse, CNRS, Toulouse, France

${ }^{2}$ Robotics and Mechatronics lab, Faculty of Electrical Engineering, Mathematics \& Computer Science, University of Twente, Enschede, The Netherlands

${ }^{3}$ Autonomous Systems Lab, Department of Mechanical and Process Engineering, ETH Zurich, 8092 Zürich, Switzerland

This work was partially funded by the European Union's Horizon 2020 research and innovation programme under grant agreement ID: 871479 AERIAL-CORE

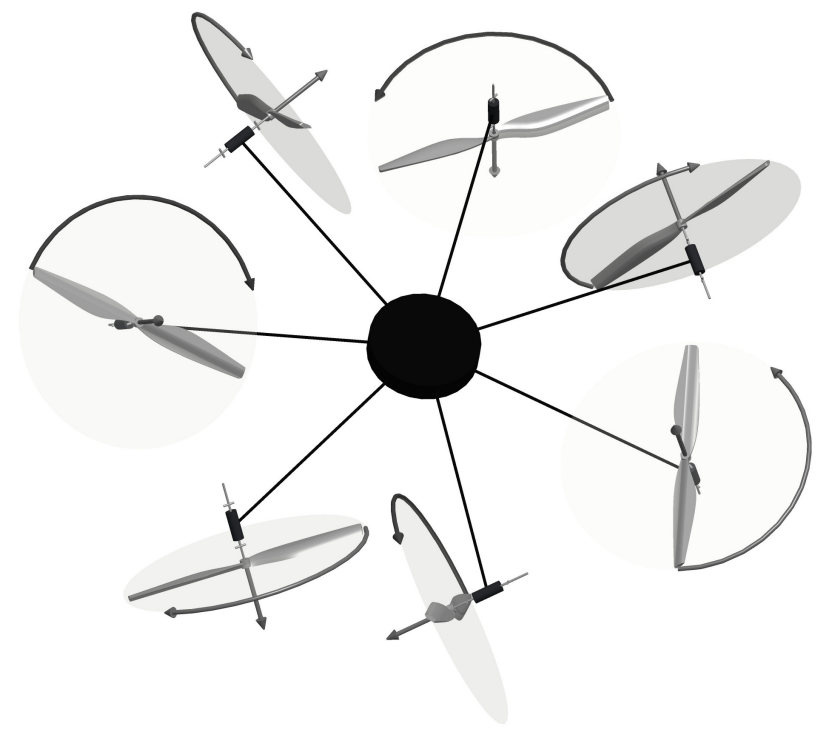

Fig. 1: Schematic of a heptarotor design with fixed uni-directional thrusters, with all propellers placed in the same plane as the platform's Center of Mass.

omnidirectional design with fixed propellers was presented in [5] and [6], achieving omnidirectionality with six bidirectional propellers and with eight uni-directional propellers, respectively. Both designs consist of a longitudinal bar, around which the propellers are oriented in an optimized direction that guarantees the omnidirectional property of the platform, while minimizing the aerodynamic interaction between adjacent propellers. Similarly, the design in [7] optimize the orientation of fixed propellers to achieve the omnidirectional property, while balancing the lift among the different propellers, and aiming for a sphere like shape of the corresponding force set. [1] later presented a working prototype of the design, where the platform employed a minimal number of uni-directional thrusters (seven) to achieve omnidirectional position and orientation tracking.

On the other hand, [8] relied on a platform with propellers actively tilting to achieve omnidirectionality. [8] presented an omnidirectional platform, consisting of a hexarotor design with each propeller rotating about its radial axis. Another version of their design was presented in [9] in which the authors placed two propellers rotating in opposite directions on each rotation axis to increase the platform efficiency, payload capacity, in addition to an increased force capacity along different directions

In the scope of omnidirectional platform design, in this 
paper we introduce novel metrics that reflect a platform's omnidirectional capability. These are calculated numerically from the platform's geometry and actuation limits. While each of the reviewed omnidirectional designs could achieve the omnidirectional property with a different actuation approach, the presented metrics reflect this property for any MRAV, while taking into consideration the platform's design and actuation limits. We then demonstrate the use of the presented metrics to analyze the omnidirectional property of "Omni-plus-seven", the design presented in [1], and of a revised version of it with similar geometry and different weight and actuation properties (see Fig. 1.)

As such, the rest of this paper is organized as follows: section II models a generic multi-rotor aerial vehicle, and presents the metrics required to analyze the corresponding omnidirectional properties; section III analyzes the omnidirectional property of the design from [1], and shows the application of the presented metrics to design a similar platform with different dimensions. Section IV concludes the paper.

\section{TECHNICAL BACKGROUND}

\section{A. Modeling of Multi-Rotor Aerial Vehicles}

Let us first define a world frame $\mathscr{F}_{W}$ with origin $O_{W}$ and axes $\left\{\boldsymbol{x}_{W}, \boldsymbol{y}_{W}, \boldsymbol{z}_{W}\right\}$ following the East-North-Up (ENU) convention. In this frame we define a generic multirotor aerial vehicle as an aerial vehicle actuated by a group of $N$ propellers placed around the platform's CoM. Let us define a frame $\mathscr{F}_{R}$ with origin $O_{R}$ centered at the platform's Geometric Center (GC) assumed to coincide with the platform's CoM, with axes $\left\{\boldsymbol{x}_{R}, \boldsymbol{y}_{R}, \boldsymbol{z}_{R}\right\}$. Each propeller is assumed to provide thrust only along its axis, which could be either fixed or actuated. Furthermore, let us define $\boldsymbol{p}_{R} \in \mathbb{R}^{3}$ and $\boldsymbol{R}_{R} \in S O(3)$ as the position of $O_{R}$ in $\mathscr{F}_{W}$ and orientation of $\mathscr{F}_{R}$ with respect to (w.r.t.) $\mathscr{F}_{W}$. Let us also define $\boldsymbol{v}_{R} \in \mathbb{R}^{3}$ as the translational velocity of $O_{R}$ in $\mathscr{F}_{W}$, and $\omega_{R} \in \mathbb{R}^{3}$ as the angular velocity of $\mathscr{F}_{R}$ w.r.t. $\mathscr{F}_{W}$ expressed in $\mathscr{F}_{R}$. Then we can write the equations of motion of the platform following the Newton-Euler formalism as follows:

$$
\begin{aligned}
\dot{\boldsymbol{p}}_{R} & =\boldsymbol{v}_{R} \\
\dot{\boldsymbol{R}}_{R} & =\boldsymbol{R}_{R} \Omega_{R} \\
{\left[\begin{array}{c}
m_{R} \dot{\boldsymbol{v}}_{R} \\
\boldsymbol{J}_{R} \dot{\boldsymbol{\omega}}_{R}
\end{array}\right] } & =-\left[\begin{array}{c}
g m_{R} \boldsymbol{e}_{3} \\
\boldsymbol{\omega}_{R} \times \boldsymbol{J}_{R} \boldsymbol{\omega}_{R}
\end{array}\right]+\boldsymbol{G} \boldsymbol{w}(\boldsymbol{u}),
\end{aligned}
$$

where $m_{R} \in \mathbb{R}_{>0}$ and $\boldsymbol{J}_{R} \in \mathbb{R}_{>0}^{3 \times 3}$ are correspondingly the platform mass and inertia matrix expressed in $\mathscr{F}_{R}, \boldsymbol{u}$ represents the control inputs that control the thrust intensity and direction of the propellers, $\boldsymbol{w}=\left[\boldsymbol{f}^{\top} \boldsymbol{m}^{\top}\right]^{\top} \in \mathbb{R}^{6}$ is the total wrench applied on the platform w.r.t. $\mathscr{F}_{R}$, and finally $\boldsymbol{G}$ brings $\boldsymbol{f}$ into the world frame as follows:

$$
\boldsymbol{G}=\left[\begin{array}{cc}
\boldsymbol{R}_{R} & \mathbf{0}_{3} \\
\mathbf{0}_{3} & \boldsymbol{I}_{3}
\end{array}\right]
$$

Let us define the full allocation matrix as the relation between the change in platform inputs and the change in the applied wrench as follows:

$$
\boldsymbol{F}(\boldsymbol{u})=\frac{\partial \boldsymbol{w}}{\partial \boldsymbol{u}}=\left[\begin{array}{ll}
\frac{\partial f}{\partial \boldsymbol{u}_{\lambda}}\left(\boldsymbol{u}_{\boldsymbol{V}}\right) & \frac{\partial \boldsymbol{f}}{\partial \boldsymbol{u}_{V}}(\boldsymbol{u}) \\
\frac{\partial \boldsymbol{m}}{\partial \boldsymbol{u}_{\lambda}}\left(\boldsymbol{u}_{\boldsymbol{V}}\right) & \frac{\partial \boldsymbol{m}}{\partial \boldsymbol{u}_{V}}(\boldsymbol{u})
\end{array}\right]
$$

where the control inputs were divided into the thrust part $\boldsymbol{u}_{\lambda} \in \mathbb{R}^{N}$ and the vectoring part $\boldsymbol{u}_{\boldsymbol{V}} \in \mathbb{R}^{m}$, where $m$ is the number of inputs that control the vectoring of all propellers on-board the platform.

In the case of fixed propellers, or propellers not actively tilting, the full allocation matrix can be made visible in (3), where the relation between the propeller thrusts and the applied wrench is linear, and can be written as follows:

$$
\begin{aligned}
\boldsymbol{w}(\boldsymbol{u}) & =\boldsymbol{F}(\boldsymbol{u})\left[\begin{array}{ll}
\boldsymbol{u}_{\lambda} & \mathbf{0}
\end{array}\right]^{\top} \\
& =\left[\begin{array}{ll}
\frac{\partial \boldsymbol{f}}{\partial \boldsymbol{u}_{\lambda}}\left(\boldsymbol{u}_{\boldsymbol{V}}\right) & \frac{\partial \boldsymbol{f}}{\partial \boldsymbol{u}_{V}}(\boldsymbol{u}) \\
\frac{\partial \boldsymbol{m}}{\partial \boldsymbol{u}_{\lambda}}\left(\boldsymbol{u}_{\boldsymbol{V}}\right) & \frac{\partial \boldsymbol{m}}{\partial \boldsymbol{u}_{\boldsymbol{V}}}(\boldsymbol{u})
\end{array}\right]\left[\begin{array}{c}
\boldsymbol{u}_{\lambda} \\
\mathbf{0}
\end{array}\right] .
\end{aligned}
$$

On the other hand, in the case of propellers actively rotation, (3) needs to be derived one more time to make appear the full allocation matrix as follows:

$$
\begin{aligned}
{\left[\begin{array}{c}
m_{R}^{W} \dddot{\boldsymbol{p}}_{B} \\
\boldsymbol{J}^{B} \ddot{\boldsymbol{\omega}}_{B}
\end{array}\right]=} & \boldsymbol{g}\left({ }^{W} \dot{\boldsymbol{p}}_{B},{ }^{W} \boldsymbol{R}_{B}, \boldsymbol{\omega}_{B}{ }^{B} \boldsymbol{P}, \boldsymbol{u}_{\boldsymbol{V}}\right) \\
& +\mathbf{G} \boldsymbol{F}(\boldsymbol{u}) \dot{\boldsymbol{u}}
\end{aligned}
$$

where $\boldsymbol{P}$ groups the position of all propellers, and $\boldsymbol{g}\left({ }^{W} \dot{\boldsymbol{p}}_{B},{ }^{W} \boldsymbol{R}_{B}, \boldsymbol{\omega}_{B}{ }^{B} \boldsymbol{P}, \boldsymbol{u}_{\boldsymbol{V}}\right)$ gathers all the terms that do not depend on $\dot{\boldsymbol{u}}$. As such, the full allocation matrix describes how the input variance affects the applied wrench, and the corresponding platform motion. We refer the interested reader to [2] for more details concerning the limitations and assumptions of this model.

\section{B. Omnidirectional Capability}

In what follows we will summarize the mathematical conditions necessary for a platform to achieve omnidirectional hoverability from [2]. After, we will complement them with the conditions for a platform to achieve the omnidirectional capability given its weight and actuation limits.

Any hovering ability of a platform relies on its ability to apply forces in certain directions while controlling its orientation. As such, any hovering ability can be deduced from the platform's wrench set $\mathbb{W}$, which can be deduced from the number of control inputs $n_{u}$, the set of feasible inputs $\mathbb{U}$, and the full allocation matrix $\boldsymbol{F}$.

In this context, we define an omnidirectional platform as a platform that can apply forces in a sphere around the origin of its force set, while applying zero-moment. Formally, an omnidirectional platform is a platform that has the following properties:

Property 1. The platform's total moment can be varied in any direction of $\mathbb{R}^{3}$, i.e.,

$$
\operatorname{rank}\left\{\frac{\partial \boldsymbol{m}}{\partial \boldsymbol{u}}\right\}=3
$$


Property 2. The platform can apply non-zero force while applying zero-moment, i.e.,

$$
\operatorname{int}\{\mathbb{W}\} \ni\left[\begin{array}{l}
\bar{f} \\
\mathbf{0}
\end{array}\right] \neq\left[\begin{array}{l}
\mathbf{0} \\
\mathbf{0}
\end{array}\right] .
$$

Property 3. The platform control is fully actuated (FA), i.e.,

$$
\operatorname{rank}(\boldsymbol{F})=6 .
$$

Finally, the omnidirectional property can be deduced from the attainable wrench set $\mathbb{W}$, where

Property 4. a platform is omnidirectional under the following conditions:

$$
\text { FA and }\left[\begin{array}{l}
\mathbf{0} \\
\mathbf{0}
\end{array}\right] \in \operatorname{int}\{\mathbb{W}\} \text {. }
$$

Note that the above definitions do not take into consideration the ability of the platform to lift its weight in different directions; as such, in what follows we will present novel metrics relying on the force set analysis of the platform to better understand its omnidirectional capability. Conditions presented hereafter are complementary to Properties 1 to 4 .

\section{Force set analysis}

Let us first define the force set of a platform (or the force set at hover), noted as $\mathscr{F}_{1}$, as the set of forces the platform could apply while applying zero-moment. Formally, we define $\mathbb{U}_{1}$ as the set of control inputs that guarantee zeromoment as follows:

$$
\mathbb{U}_{1}=\left\{\boldsymbol{u} \in \mathbb{U} \mid \boldsymbol{w}(\boldsymbol{u})=\left[\begin{array}{l}
\tilde{f} \\
\mathbf{0}
\end{array}\right]\right\},
$$

for any feasible force $\overline{\boldsymbol{f}}$.

Following the definition of $\mathbb{U}_{1}$, we can define $\mathscr{F}_{1}$ as the image of $\mathbb{U}_{1}$ as follows:

$$
\mathscr{F}_{1}=\left\{\boldsymbol{y} \in \mathbb{R}^{3} \mid \boldsymbol{u} \in \mathbb{U}_{1}, \boldsymbol{w}(\boldsymbol{u})=\left[\begin{array}{l}
\boldsymbol{y} \\
\mathbf{0}
\end{array}\right]\right\} .
$$

We refer to [2] for a detailed method of the computation of the $\mathscr{F}_{1}$ set for different MRAV platforms.

It is easy to see that, following Property 4, a platform is omnidirectional if the origin is an interior point of $\mathscr{F}_{1}$. However, in addition to this condition, an omnidirectional platform should be able to apply a force in any direction enough to counteract its weight. As such, we amend Property 4 with the following condition:

Proposition 1. A platform is omnidirectional if it is fully actuated and it can lift its weight in any direction about the origin, i.e.,

$$
\text { FA and } \forall \overline{\boldsymbol{f}} \in \operatorname{ball}_{\mathbb{R}^{3}}\left(\left\|m_{R} * \boldsymbol{g}\right\|\right), \overline{\boldsymbol{f}} \ni \mathscr{F}_{1}
$$

As such, and given the $\mathscr{F}_{1}$ set of a platform, we introduce the following metrics that allow the assessment of the omnidirectional property of a platform:

- Omnidirectional Lift (ODL): this metric reflects the lift that could be applied in any direction. Mathematically, it can be computed as the radius of the maximum inscribed sphere in $\mathscr{F}_{1}$, centered at the $\mathbf{0}$ of the set.
- Multi-Directional Lift (MDL): this metric reflects the maximum lift that could be applied along at least one direction. Mathematically, it can be computed as the radius of the minimum circumscribed sphere about $\mathscr{F}_{1}$, centered at the $\mathbf{0}$ of the set.

Following proposition 1, and the definition of ODL, we can deduce the following:

\section{Corollary 1. A platform is omnidirectional iff}

$$
\text { FA and } \mathrm{ODL} \geq m_{R} g
$$

On the other hand, we define a partial-omnidirectional platform as follows:

Proposition 2. a partial omnidirectional platform is a platform such that

$$
\begin{aligned}
& \mathrm{MDL} \geq m_{R} g \geq \mathrm{ODL} \\
& \text { FA and }\left[\begin{array}{l}
\mathbf{0} \\
\mathbf{0}
\end{array}\right] \in \operatorname{int}\{\mathbb{W}\}
\end{aligned}
$$

Note that a partially omnidirectional platform can statically hover in one or more directions, and might be able to dynamically hover in other directions (we refer readers to [2] for the definition of dynamic hovering, and to [10] for an example MRAV that can achieve dynamic hovering; note that the platform in [10] is underactuated and as such, it serves in the scope of this paper to demonstrate the concept of dynamic hovering and not the concept of partially omnidirectional platforms).

As such, and following the above two definitions, a platform that is fully actuated and satisfying property 4 could be classified in one of the following three categories based on its actuation limits and weight:

- if ODL $\geq m_{R} g$, the platform is fully omnidirectional. This is the case of any platform that could lift its weight in all directions while apply zero-moment.

- if MDL $\geq m_{R} g \geq \mathrm{ODL}$, the platform is partially omnidirectional. This is the case of any platform that is able to apply forces in all directions while applying zeromoment, however, which can lift its weight in at least one direction but not all directions. One visual example can be seen in a variant of the plot in Fig. 2: if the force set of a platform has the same shape of the one in this figure, with the inscribed sphere smaller than the platform's $m_{R} g$, and with the distance between at least one of the edges and the center of the set being larger than $m_{R} g$, then the platform is partially omnidirectional.

- if $m_{R} g>\mathrm{MDL}$, then the platform is neither fully nor partially omnidirectional. This is the case of any platform that could apply forces in all directions while applying zero-moment, however, which has an elevated weight as compared to its actuation limits. Note that in this case, the platform cannot lift its weight in any direction, and as such cannot statically hover. 

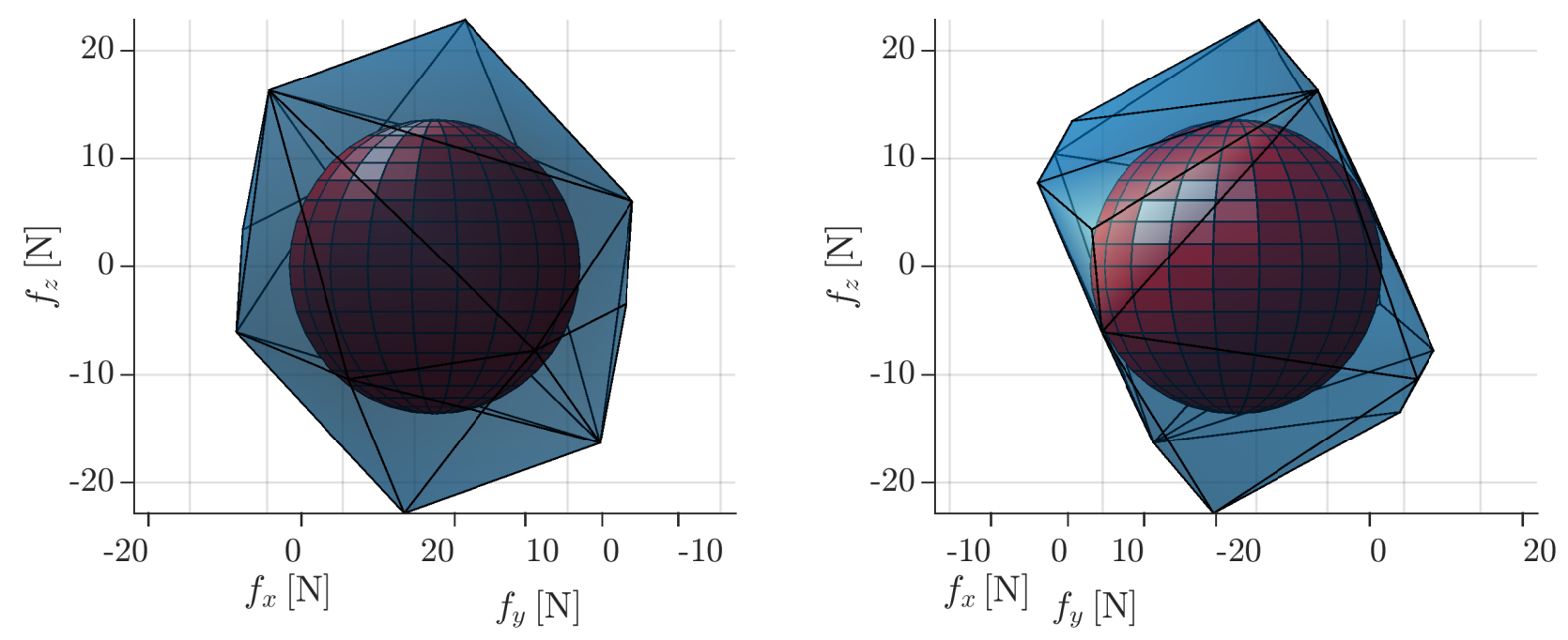

Fig. 2: Two side views of the $\mathscr{F}_{1}$ set of the platform presented in [1], with the corresponding sphere showing the ODL metric.

\section{OMNIHEPTAROTOR WITH UNI-DIRECTIONAL THRUSTERS}

This section analyzes the omnidirectional capability of the design presented in [1], [7]. The platform is a heptarotor with 7 fixed uni-directional propellers. The propeller positions are chosen by the authors, while their orientation is optimally chosen to ensure an equal sharing of thrust along the different thrusters, and to ensure a spherical shape of the force/moment set. The presented prototype in [1] is a small vehicle with a distance between propellers' CoM and the platform's CoM of $d=0.16[\mathrm{~m}]$, and a weight of about $m_{R}=1.1[\mathrm{Kg}]$. The used identical thrusters have a drag to lift coefficient $c=0.002$ [m], and a lift coefficient $c_{f}=0.5 e^{-4}\left[\mathrm{~N} / \mathrm{Hz}^{2}\right]$, and can generate a maximum lift $u_{\max }=14[\mathrm{~N}]$. We omit the details concerning the propeller orientations for the compactness of this paper, and we show in Fig. 2 the $\mathscr{F}_{1}$ set of the corresponding platform, and the sphere corresponding to the ODL metric.

It can be seen from this figure that while the design was optimized to guarantee that this platform can apply forces equally in all directions, the inscribed sphere touches the force set from one side, while allowing larger forces to be applied in other directions. This discrepancy between the maximum force applied in different directions can also be seen from the presented metrics, where the given platform has an $\mathrm{ODL}=1.37[\mathrm{Kg}]$ (normalized by $g$ ), and an $\mathrm{MDL}=2.34[\mathrm{Kg}]$ (normalized by $g$ ). These metrics also show that the built platform can lift its weight in any direction, and as such, it is a fully omnidirectional platform.

\section{A. Upgraded prototype}

As mentioned in [1], the presented heptarotor prototype has a few drawbacks; one of the mentioned drawbacks is the high propeller velocity required to achieve $u_{\max }$ per thruster. Such high velocity can cause human discomfort if the platform is eventually operated in the proximity of a human, in addition to the increased danger it could cause, and the possibility to reach actuation limits while allocating the required wrench. As such, in what follows we will study the possibility of designing an improved prototype that requires lower propeller rotational speeds.

To be able to achieve the new design, we aim first to build a platform with larger propellers so as to produce a larger thrust per propeller. We will focus in this part on a platform with $d=0.4[\mathrm{~m}]$ similar to the one presented in [11], where as we will show later, the larger arm length is a necessity to place larger propellers on-board the platform, without any collision between adjacent propellers. Following the design from [11], we can estimate the platform weight with 7 motors and propellers at $m_{R} \approx 2.4[\mathrm{Kg}]$. This estimate assumes that we use similar structural parts and core electronics (processing unit and sensors), while motors are replaced with MK3644 from MikroKopter due to the higher power requirement of this design. Table I summarizes the weight of the different components of this design. It should be noted that Electronic Speed Controllers should be replaced as well in the new design, since the ones used in [11] might not be able to achieve the required rotational speeds; while we do not specify the required ESCs in this paper, as it is out of the scope of the study conducted in this paper, its selection does not substantially affect the platform weight.

Figure 3 shows the ODL and MDL metrics with respect to varying $u_{\max }$. These metrics are normalized by $g$ to reflect the feasible platform mass. We can see from this figure that the relation between the mass the platform from [1] can 


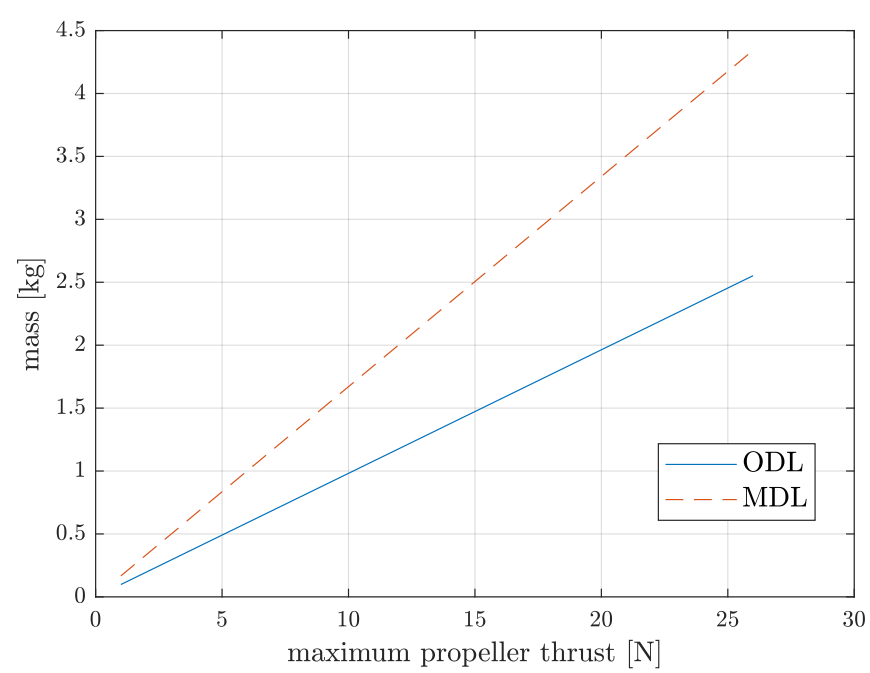

Fig. 3: Omnidirectional property metrics of the heptarotor design from [1] for different maximum thrust per propeller.

\begin{tabular}{lccc}
\hline Component & weight per unit $[\mathrm{g}]$ & \# units & weight $[\mathrm{g}]$ \\
\hline Motors & 111 & 7 & 777 \\
Propellers & 15 & 7 & 105 \\
Motor Mountings & 22 & 7 & 154 \\
Electronics & 391 & - & 391 \\
Mechanical parts & 549 & - & 549 \\
Battery & 398 & - & 398 \\
\hline Total & & & 2374 \\
\hline
\end{tabular}

TABLE I: $\mathrm{O}_{+}^{7}$ estimated new design: weight estimatation of each of the platform components based on the components used for the TiltHex platform ( [11]).

lift and its $u_{\max }$ is linear. As such, we estimate that for the suggested design $\left(m_{R}=2.4[\mathrm{Kg}]\right)$, the required maximum lift per propeller is $u_{\max }=24.5[\mathrm{~N}]$.

To estimate the maximum propeller size that could be placed on such a platform, let us note that the design in [1] places all propellers in the same plane as the platform's CoM, at an equal distance $d$ from the $\mathrm{CoM}$, and with an equal distance between adjacent propellers. As such, the maximum propeller diameter that can be placed in such design is equal to the circle cord between two adjacent propeller positions. Equivalently, $\max$ diameter $=2 d \sin \left(\frac{\pi}{7}\right)$. As such, for the corresponding proposed platform, the maximum diameter is 13.7". In order to avoid any collision between the tips of adjacent propellers, and to be able to use off the shelf propellers, we will focus in our analysis on 13" propellers, with a lift coefficient $c_{f}=12.19 \times 10^{-4}\left[\mathrm{~N} / \mathrm{Hz}^{2}\right]$. As such, the required maximum propeller rotational speed is $\omega_{\max }=142[\mathrm{~Hz}]$, which is much lower than the rotational speed required by the prototype in [1] $\left(\omega_{\max }=432[\mathrm{~Hz}]\right)$.

To be able to increase further the propeller size (and subsequently reduce the required maximum rotational speed), we study the placement of all propellers equally spaced on a sphere around the platform's CoM. The placement of the propellers is done in a way to maximize the distance between adjacent propellers using the Bauer's spiral method [12],

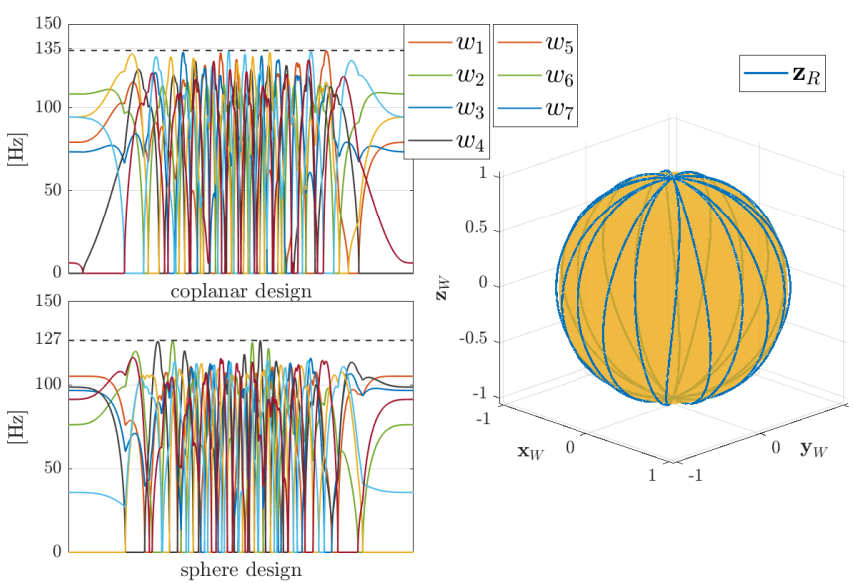

Fig. 4: showing on the left top the required propeller commands for the coplanar design to hover in different directions, and bottom the required propeller commands for the sphere design to hover in different directions. The desired directions are such that the platform's $z_{R}$ rotates about a unit sphere as shown in figure on the right.

and the minimum distance is computed numerically. Following this method, the maximum propeller diameter is such that the maximum diameter is 15.75 ". As we did with the planar placement case, let us focus on 15" propellers to avoid collision between adjacent propellers and to be able to use off the shelf propellers. These propellers can achieve a lift coefficient $c_{f}=14.41 \times 10^{-4}\left[\mathrm{~N} / \mathrm{Hz}^{2}\right]$, and as such, the required maximum propeller rotational speed is reduced to $\omega_{\max }=130[\mathrm{~Hz}]$. It should be noted that while the spherical placement reduces the maximum required propeller rotational speed, the design is more complex to conceive than the planar one.

\section{B. Optimized Design}

Following the above calculations, we optimize the propeller orientation for the chosen dimensions in the case of the coplanar and the sphere designs following the algorithm from [7]. A schematic of the two designs are shown respectively in Fig. 1 and Fig. 5. To validate the findings of subsection III-A, in Fig. 4 we show the required propeller commands for each of the two optimized platforms to hover in different directions about their respective CoMs. These directions are such that the platform would hover with its vertical axis rotating around a unit sphere as shown in Fig. 4. This figure shows that the required maximum propeller command for each platform is lower than the maximum propeller command required by the design in [1], and lower than the expected one in subsection III-A. This shows that the design technique shown above can decrease the required propeller speeds of the platform, while at the same time achieving the omnidirectional property. Moreover, this figure also shows that the optimized design could achieve maximum propeller speeds that are lower than the estimated ones, where the estimation was done with a platform with fixed propeller orientations, irrespective of its dimensions. 


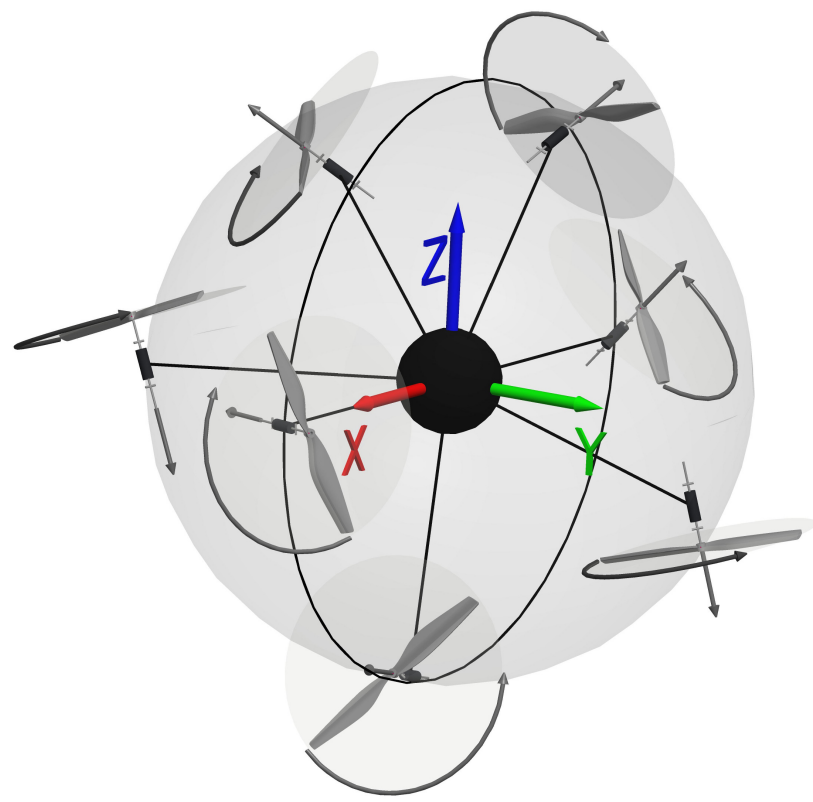

Fig. 5: Schematic of a heptarotor design with fixed uni-directional thrusters, with propellers equally spaced on a sphere centered in the platform's Center of Mass.

\section{CONCLUSION}

In this paper we presented novel metrics to assess the omnidirectional property of a generic multi-rotor aerial vehicle. These metrics are calculated numerically from the force set of the platform, and as such rely on the platform geometry and thrusters; moreover, they allow a direct assessment of a platform's omnidirectional property given its weight.

Following the definition of these metrics, we showed a direct application by assessing the omnidirectional property of the novel heptarotor with fixed uni-directional thrusters presented in [1].

In addition, we showed the use of these metrics in the design process of an upgraded design of the above mentioned heptarotor. In this process, these metrics allowed us to choose the corresponding thrusters, and calculate the required propeller speeds to achieve the omnidirectionality of the desired design, given its geometry and estimated weight. We also showed the same design process when propellers are placed in the same plane of the platform's CoM, and when placed in a sphere around the platform's CoM. In the future, we aim to build the designed platform, where as mentioned above the electronics required for the platform flight still need to be chosen. In addition, it would be interesting to design the platform with lighter material to be able to achieve the omnidirectional property with lighter actuators.

\section{REFERENCES}

[1] M. Hamandi, K. Sawant, M. Tognon, and A. Franchi, "Omni-plusseven $\left(\mathrm{O}_{+}^{7}\right)$ : An omnidirectional aerial prototype with a minimal number of uni-directional thrusters," in 2020 Int. Conf. on Unmanned Aircraft Systems, Athens, Greece, Sep. 2020.
[2] M. Hamandi, F. Usai, Q. Sable, N. Staub, M. Tognon, and A. Franchi, "Design of multirotor aerial vehicles: a taxonomy based on input allocation," accepted, The International Journal of Robotics Research, 2021.

[3] A. Ollero, M. Tognon, A. Suarez, D. J. Lee, and A. Franchi, "Past, present, and future of aerial robotic manipulators," IEEE Trans. on Robotics, 2021.

[4] D. Brescianini and R. D'Andrea, "Design, modeling and control of an omni-directional aerial vehicle," in 2016 IEEE Int. Conf. on Robotics and Automation, Stockholm, Sweden, may 2016, pp. 3261-3266.

[5] S. Park, J. Her, J. Kim, and D. Lee, "Design, modeling and control of omni-directional aerial robot," in 2016 IEEE/RSJ Int. Conf. on Intelligent Robots and Systems, Daejeon, Korea, oct 2016, pp. 15701575.

[6] S. Park, J. Lee, J. Ahn, M. Kim, J. Her, G. H. Yang, and D. Lee, "Odar: Aerial manipulation platform enabling omni-directional wrench generation," IEEE/ASME Transactions on Mechatronics, vol. 23, no. 4 pp. 1907-1918, 2018.

[7] M. Tognon and A. Franchi, "Omnidirectional aerial vehicles with unidirectional thrusters: Theory, optimal design, and control," IEEE Robotics and Automation Letters, vol. 3, no. 3, pp. 2277-2282, 2018

[8] M. Kamel, S. Verling, O. Elkhatib, C. Sprecher, P. Wulkop, Z. Taylor, R. Siegwart, and I. Gilitschenski, "The voliro omniorientational hexacopter: An agile and maneuverable tiltable-rotor aerial vehicle," IEEE Robotics \& Automation Magazine, vol. 25, no. 4, pp. 34-44, 2018.

[9] M. Allenspach, K. Bodie, M. Brunner, L. Rinsoz, Z. Taylor, M. Kamel, R. Siegwart, and J. Nieto, "Design and optimal control of a tiltrotor micro-aerial vehicle for efficient omnidirectional flight," The International Journal of Robotics Research, vol. 39, no. 10-11, pp. 1305$1325,2020$.

[10] P. Rongier, E. Lavarec, and F. Pierrot, "Kinematic and dynamic modeling and control of a 3-rotor aircraft," in 2005 IEEE Int. Conf. on Robotics and Automation, Barcelona, Spain, april 2005, pp. 26062611.

[11] M. Ryll, G. Muscio, F. Pierri, E. Cataldi, G. Antonelli, F. Caccavale, and A. Franchi, "6D physical interaction with a fully actuated aerial robot," in 2017 IEEE Int. Conf. on Robotics and Automation, Singapore, May 2017, pp. 5190-5195.

[12] M. K. Arthur, "Point picking and distributing on the disc and sphere," Tech. Rep., 2015 